\title{
EFFECTS OF U-SHAPED TWO-STEP THROTTLING GROOVE PARAMETERS ON CAVITATION EROSION CHARACTERISTICS ${ }^{1}$
}

\author{
Wenhua Jia, Yanyan LiU \\ School of Mechanical Engineering, Nanjing Institute of Technology, Jiangsu, China \\ e-mail: geovrml@163.com (WenhuaJia) \\ Chenbo Yin \\ School of Mechanical and Power Engineering, Nanjing University of Technology, Jiangsu, China \\ Guo Li, Dasheng Zhu, Shen Ding \\ School of Mechanical Engineering, Nanjing Institute of Technology, Jiangsu, China
}

\begin{abstract}
Throttling usually occurs when a fluid passes through an orifice, sometimes even severe cavitation erosion may occur. In this study, the equation for the cavitation index of a throttling valve was proposed and the cavitation erosion area in the throttle valve was found to change its position with the orifice opening $(X)$. Cavitation features of singular and two-port series throttling grooves were characterized by defining cavitation indexes $\sigma_{1}$ and $\sigma_{2}$, because the cavitation index- $\sigma$ can determine the occurrence and intensity of cavitation. Then the indexes $\sigma_{1}$ and $\sigma_{2}$ included internal geometric parameters and external pressure boundaries were obtained, and cavitation indexes curves $\sigma_{1}-X$ and $\sigma_{2}-X$ were also plotted. From the curves of the cavitation index, it was observed that cavitation concentration section also would transfer with opening $X$ changes in the U-shaped groove. The depth of the U-shaped groove had a more evident impact on cavitation, whereas the effect of width on cavitation erosion was not so obvious. The intensity of cavitation erosion when the fluid flowed into the orifice section of the U-shaped groove was always larger than that when the fluid flowed away.
\end{abstract}

Keywords: cavitation erosion; throttling groove; distribution coefficient

\section{Introduction}

Any hydraulic oil inevitably contains impurity gases (Gnanaskandan and Mahesh, 2016), and when the pressure difference in a certain area is large (Karrholm et al., 2007) the dissolved gas will be separated from the oil and becomes bubbles (Koukouvinis et al., 2017), resulting in cavitation (Chen et al., 2019). Cavitation bubbles will become compressed and then collapse, leading to high temperatures in some areas and destroying the inner and outside surfaces of the valve (Liu et al., 2019). The damage that cavitation does to the valve is often due to the high-temperature and high-pressure area that forms in the region where the collapse of cavitation bubbles takes place (Ghiji et al., 2017). Amirante et al. (2014) found that when the cavitation bubbles swelled and collapsed around the contracted section of the throttle valve, which was also known as the minimum flow section, the explosion energy was usually toward the valve wall, causing great damage to the valve. Sun et al. (2020) also found that harsh noise was associated with the collapse of bubbles. Kim and Kim (2019) discovered an instantaneous change in the pressure of the bubbles when the collapse occurred. Požar et al. (2020) analyzed the cavitation pattern, vibration and noise in a typical hydraulic orifice through experiments,

\footnotetext{
${ }^{1}$ Presented paper from the 4th International Conference on Material Strength and Applied Mechanics, MSAM 2021
} 
and found that the periodical fall-off of cavitation and cavitation had a spiral pattern with length changing periodically with time. Favrel et al. (2019) investigated the flow field characteristics for a two-phase flow in different orifices and derived the theoretical pressure dropped for the fluid passing through the orifice, which were validated by an experiment and numerical simulation. Yaghoubi et al. (2018) applied the combination of the RNG $k$ - $\xi$ turbulence model and multi-phase flow technique to numerical simulations of the cavitation flow field of a ball throttle valve. Han et al. (2017) carried out noise testing and spectral analysis in a butterfly valve with and without cavitation, and the result showed an increment in the noise level of the butterfly valve under cavitation. Jiang et al. (2015) found that despite different flow parameters, cavitation was always initiated near the sharp-edged orifice, which was at the intersection of the throttling edge and hole wall, and the initial location would change slightly with the orifice opening $X$. Spectral analysis demonstrated that the shape and size of cavitation were the most critical factors affecting throttling noise in the orifice.

The cavitation index $\sigma$ is an important index that determines the occurrence and intensity of cavitation (Corchuelo and Soto, 2017), which is usually denoted by $\sigma$ indicating the significance of the cavitation probability of a hydraulic orifice. Liu et al. (2020) studied the effect of the jet angle on cavitation by using a CFD method under different cavitation numbers. Šarc et al. (2017) compared the cavitation numbers of different geometric shapes, and transformed the general $\sigma$ of the U-shaped groove into specific $\sigma$, which is helpful to study the relationship between structural parameters of the U-shaped groove and cavitation erosion. It is very necessary to calculate the cavitation number of different throttle sections so that the possibility of cavitation in different sections could be characterized in order to study the cavitation characteristics of the throttling groove valve. Therefore, in this study, changes in the pressure and velocity of a hydraulic oil when it passed through the U-shaped groove were analyzed, and a low pressure zone where possible cavitation erosion occurred was found to be in the proximity of the groove. Then, the exact expression of $\sigma$ representing the cavitation characteristics in the U-shaped groove orifice was derived from the general equation for $\sigma$. Additionally, with the establishment of the $\sigma-X$ curves in flow sections, the location of the intensive throttle valve cavitation zone was found to vary with $X$, and the effects of groove depth, groove radius and flow direction on cavitation were then investigated.

\section{Modeling and simulation of the flow field characteristics of the U-shaped groove}

The structural diagram of the large cavity structure of a U-shaped groove was shown in Fig. 1, where $c$ is the diameter of the inlet, $c=10 \mathrm{~mm} ; b$ is the distance between the inlet and outlet, $b=12 \mathrm{~mm}$; $a$ is the diameter of outlet, $a=13 \mathrm{~mm}$; $d$ is the diameter of the main valve core rod, $d=18 \mathrm{~mm} ; D$ is the diameter of valve spool shoulder, $D=28 \mathrm{~mm} ; e$ is the radius of the fillet at the valve seat, $e=5 \mathrm{~mm} ; h$ is the corner radius, $h=5 \mathrm{~mm} ; X$ is the orifice opening of the inlet of the U-shaped groove valve; $A_{1}(X)$ and $A_{2}(X)$ are the areas of the fluid flowing through throttling section I and section II, respectively:

— if $X<R, A_{1}(X)$ and $A_{2}(X)$ can be calculated by

$$
\begin{gathered}
A_{1}(X)=R_{2} \arccos \left(\frac{R-X}{R}\right)-(R-X) \sqrt{X(2 R-X)} \\
A_{2}(X)=2 H \sqrt{X(2 R-X)} \\
\text { - if } X \geqslant R, A_{1}(X) \text { and } A_{2}(X) \text { can be calculated by } \\
A_{1}(X)=\frac{\pi R^{2}}{2}+2 R(X-R) \quad A_{2}(X)=2 R H
\end{gathered}
$$



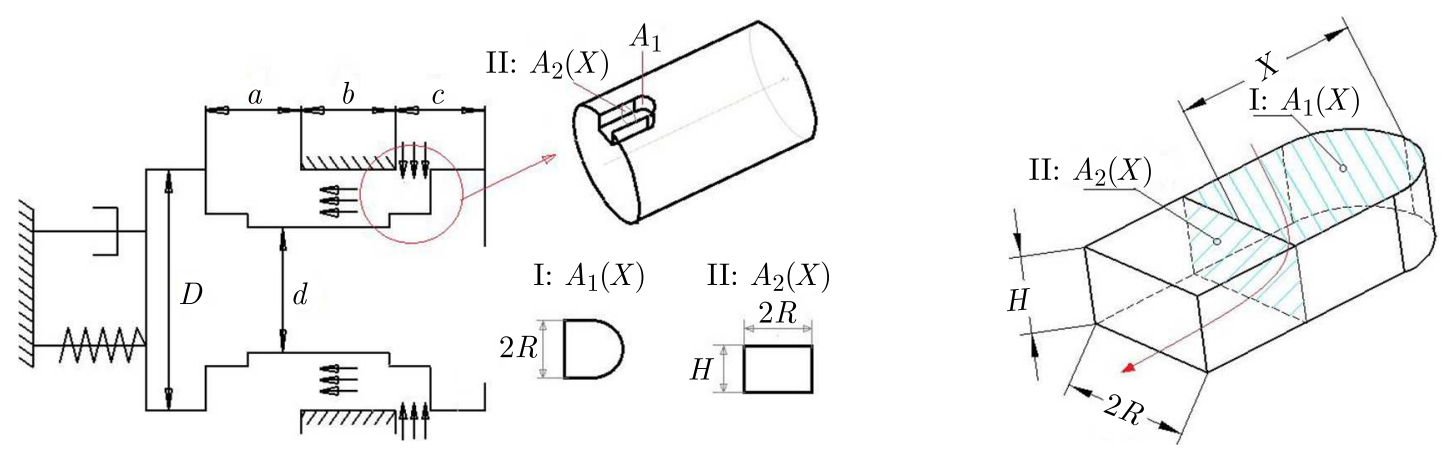

Fig. 1. Structural diagram of the U-shaped throttle groove valve

The chamber is $3 \mathrm{D}$ symmetric. Thus, the computational domain is limited by a one-quarter chamber including a one-quarter notch only, shown in Fig. $2 \mathrm{a}$. Figure $2 \mathrm{~b}$ provides a view of the unstructured (the complex notch geometry does not allow the use of structured grids) computational grid employed, meshed by about 47000 grids with tetrahedron elements. The Gambit "size function" has been used to refine the grid in the metering area to ensure adequate grid size where the maximum velocity and pressure gradients occur. All grid points of the Jacobian are positive, that is, the grid size is positive. Both the accuracy of analysis and time taken also depend on the mesh size. Hence the optimum mesh determination is an important step in CFD analysis. In the cases of velocity gradient near-wall regions and pressure gradient near valve notches, the mesh size is bigger than that in other regions. Using Fluent software to refine mesh formation, the optimal mesh is obtained, which can significantly save the preprocessing time. Figure $2 \mathrm{~b}$ also shows the inlet, the outlet and the notch chamber, and the boundary information required which have been indicated:

(1) Inlet boundary is velocity

(2) Outlet boundary is set to $0.8 \mathrm{MPa}$

(3) Density of liquid is $889 \mathrm{~kg} / \mathrm{m}^{3}$

(4) Kinematical viscosity is $4 \cdot 10^{-5} \mathrm{~m}^{2} / \mathrm{s}$

(a)

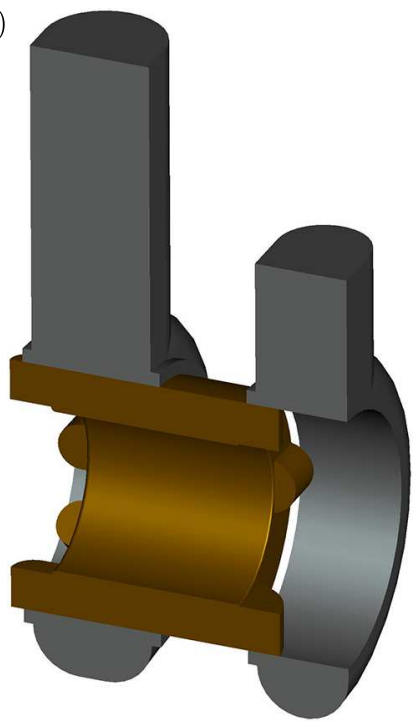

(b)

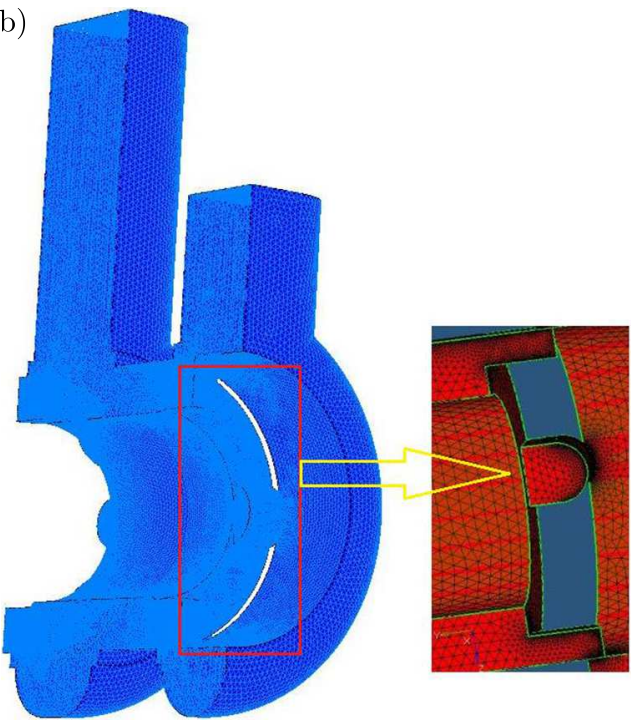

Fig. 2. (a) CFD model of the valve chamber, (b) mesh of fluid regions of the valve chamber

Since the flow field in the groove was symmetrical, a half model of the flow field at $X=3.5 \mathrm{~mm}$ was built using Fluent for hydraulic analysis, and the pressure and velocity 
nephograms when hydraulic oil flowed through the groove were shown in Figs. 3a and 4b. As shown in Fig. 3, the pressure in the inlet of the groove dropped rapidly and the velocity grew quickly forming turbulence and a low-pressure with a small range, where bubbles were very likely to form. The collapse of cavitation bubbles in the throttle valve usually results in a high speed flow and large instantaneous pressure, which is usually related to cavitation noise. And, when the orifice opening $X$ was small, the obvious turbulence can be seen, as shown in Figs. 4a and $4 \mathrm{~b}$.

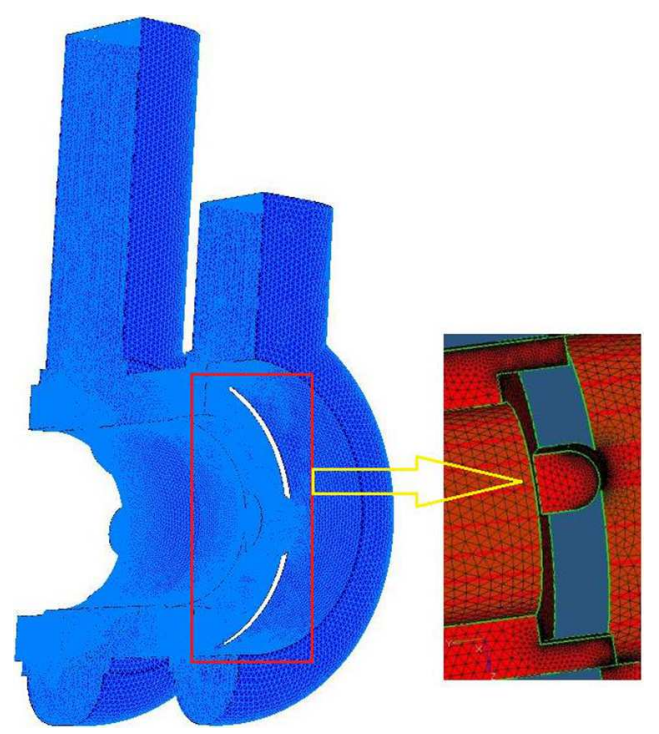

Fig. 3. The oil in the main valve chamber at $6 \mathrm{~mm}$ spool displacement: (a) pressure nephogram, (b) velocity nephogram

(a)

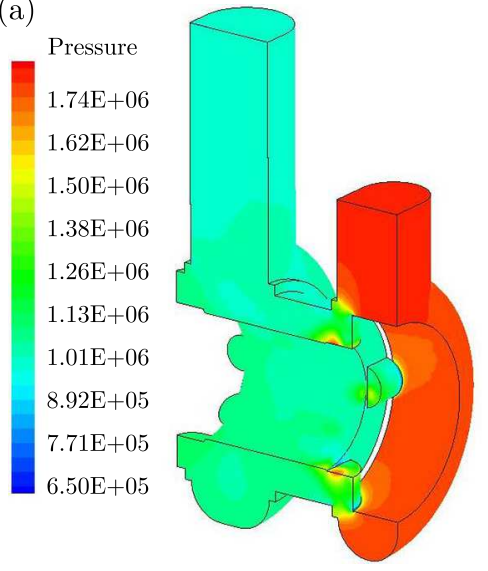

(b)

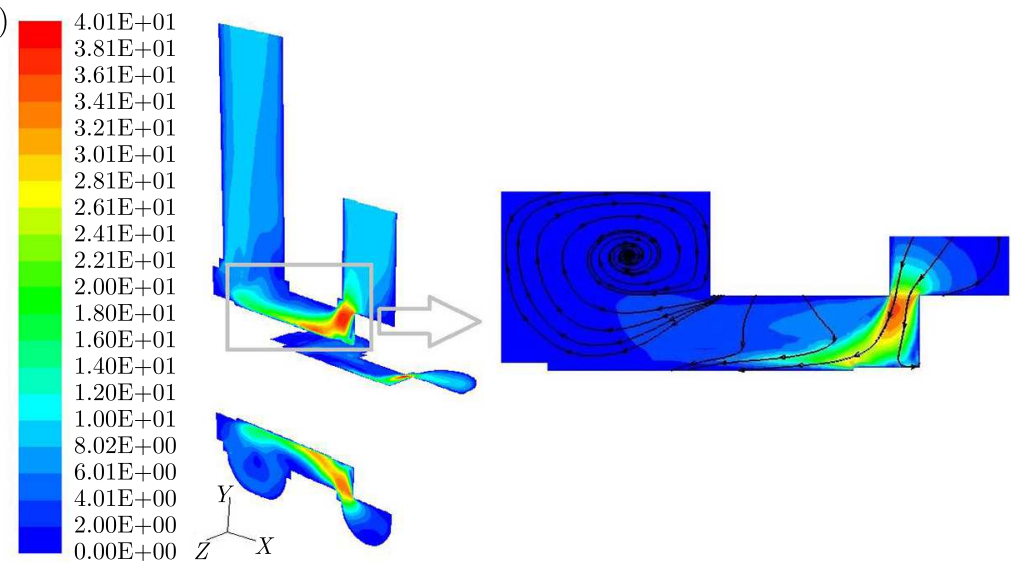

Fig. 4. (a) Diagram of velocity vector, (b) streamline diagram

\section{Analysis and discussions of cavitation erosion characteristics of the U-shaped} groove

\subsection{Deduction of the cavitation index}

The notion of a dimensionless initial value of $\sigma$ was previously proposed to quantify the condition under which cavitation of the bubbles would take place, and it implied the possibility of occurrence of cavitation bubbles under various conditions. A general definition of dimensionless $\sigma$ is given by 


$$
\sigma=\frac{P_{0}-P_{g}}{\frac{1}{2} \rho u_{0}^{2}}
$$

where $P_{0}$ is the pressure in the inlet, which is the absolute pressure of the considered fluid, $u_{0}$ is the fluid velocity, $P_{g}$ is the vapor pressure, $\rho$ is the density of the fluid (Lee et al., 2016).

According to Eq. (3.1), cavitation erosion occurs when the minimum pressure $P_{\min }$ in the specific flow field is lower than $P_{g}$.

When the minimum value of flow pressure $P_{\min }$ reaches the value of pressure $P_{g}$, the minimum pressure coefficient $C_{P_{\min }}$ is the same as the index $\sigma$, and is given by

$$
\left.C_{P_{\min }}\right|_{P_{\min }=P_{g}}=\frac{P_{g}-P_{0}}{\frac{1}{2} \rho u_{0}^{2}}=-\sigma
$$

According to Eq. (3.2), $\sigma$ under cavitation can be determined by $C_{P_{\min }}$. The larger $C_{P_{\min }}$ implies a large reduction in the pressure of the flow field, as well as a larger $\sigma$ and larger possibility of occurrence of cavitation erosion. When $C_{P_{\min }}$ falls, the pressure and $\sigma$ in the flow field will decline, thus cavitation is unlikely to happen in the flow field.

The idealized Bernoulli equation gives

$$
P_{2}-P_{0}=\frac{\rho\left(u_{0}^{2}-u_{2}^{2}\right)}{2}
$$

where $P_{2}$ is the pressure in the orifice flow section and hydraulic flow channel, $u_{2}$ and $u_{0}$ are the average fluid velocities in the orifice flow section and hydraulic flow channel. The fluid velocity in hydraulic valve passage $u_{\infty}$ and the velocity at the throttle section of the orifice $u_{2}$ have a relationship as follows

$$
\left(\frac{u_{0}}{u}\right)^{2} \ll 1
$$

Equation (3.3) is simplified to

$$
P_{0}-P_{2} \approx \frac{\rho u_{2}^{2}}{2}
$$

and

$$
\sigma=\left.C_{P_{\text {min }}}^{\prime-1}\right|_{P_{\text {min }}=P_{g}}=\frac{P_{0}-P_{2}}{P_{0}-P_{g}}
$$

According to Eq. (3.4), the cavitation index $\sigma$ of the throttling groove valve can be determined by the ratio of the pressure difference $P_{0}-P_{2}$ and $P_{0}-P_{g}$. It makes the cavitation index $\sigma$ more intuitive and easier to obtain the specific value of the cavitation index of the throttle port. In order to be more suitable for the U-shaped throttling groove of the valve, the cavitation index $\sigma$ of throttling section I and II can be calculated as $\sigma_{1}, \sigma_{2}$, respectively.

A relation between $C_{P_{\min }}^{\prime}$ of the groove orifice and $\sigma_{1}, \sigma_{2}$ is defined as

$$
\sigma_{1}=\left.C_{P_{\text {min }}}^{\prime-1}\right|_{P_{\min }=P_{g}}=\frac{P_{1}-P_{2}}{P_{1}-P_{g}}=\frac{\Delta P_{1}}{P_{1}-P_{g}}
$$

In the same way

$$
\sigma_{2}=\frac{P_{2}-P_{3}}{P_{2}-P_{g}}=\frac{\Delta P_{2}}{P_{2}-P_{g}}
$$


where $P_{1}=P_{0}$ is the pressure in the flow field at the inlet of the throttle valve, $P_{2}$ is the pressure of the flow field between two flow sections, $P_{3}$ is the pressure of the flow field at the outlet of the throttle valve, which is the pressure in the outlet. In the two-step throttle valve orifice, the overall pressure reduction should be allocated to throttling section I and II based on the principle that the magnitude is inversely proportional to the square of the flow section area $A_{2}(X)$. In other words, the pressure reduction in throttling section I and II satisfies:

$$
\frac{\Delta P_{1}}{\Delta P_{2}}=\left(\frac{C_{q 2} A_{2}(X)}{C_{q 1} A_{1}(X)}\right)^{2}=k^{2}
$$

where $C_{q 1} A_{1}(X)$ and $C_{q 2} A_{2}(X)$ are regarded as the equivalent flow sections with consideration of the processing performance of the flow sections, $k$ is the pressure reduction distribution coefficient of the two-step throttle valve orifice. The mentioned pressures were shown in Fig. 5.

(a)

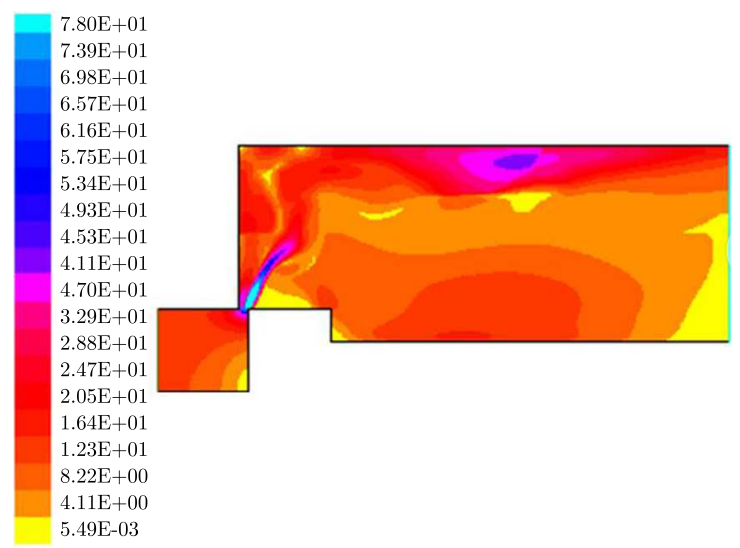

(b)

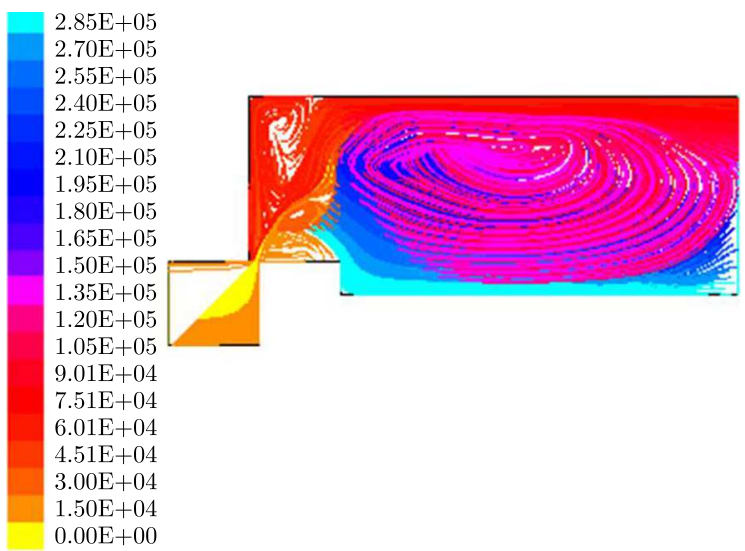

Fig. 5. Schematic diagram of each mentioned pressures

Based on the allocation principle of pressure reduction, the overall pressure reduction in the throttle valve $\Delta P_{\text {total }}$ is

$$
\Delta P_{1}+\Delta P_{2}=\Delta P_{\text {total }}
$$

Then, Eqs. (3.6) and (3.8) can be rewritten as

$$
\sigma_{1}=\frac{1-\frac{1}{\mathcal{A}_{12}}}{1-\frac{P_{g}}{P_{1}}} \quad \sigma_{2}=\frac{1-\mathcal{A}_{13} \frac{P_{2}}{P_{1}}}{1-\mathcal{A}_{13} \frac{P_{g}}{P_{1}}}
$$

where

$$
\mathcal{A}_{12}=1+\frac{k^{2}}{\left(1+\frac{1}{k^{2}}\right) \frac{1}{\frac{P_{1}}{P_{2}}-1}} \quad \mathcal{A}_{13}=1+\frac{k^{2}}{\left(1+\frac{1}{k^{2}}\right) \frac{1}{\frac{P_{1}}{P_{3}}-1}+1}
$$

Take $P_{3}$ as $0.1 \mathrm{MPa}$, since $P_{1}$ is much larger than the atmospheric pressure, $\left[\left(P_{1} / P_{3}\right)^{-1}\right]^{-1}$ in Eq. (3.10) is approximately zero, then Eq. (3.10) becomes

$$
\sigma_{1}=\frac{1-\frac{1}{1+k^{2}}}{1-\frac{P_{g}}{P_{1}}} \quad \sigma_{2}=\frac{1-\left(1+k^{2}\right) \frac{P_{3}}{P_{1}}}{1-\left(1+k^{2}\right) \frac{P_{g}}{P_{1}}}
$$

where $\sigma_{1}$ is the cavitation index of throttling section I, $\sigma_{2}$ is the cavitation index of throttling section II. According to Eq. (3.11) and the pressure reduction distribution coefficient $k$, different $\sigma$ under orifice openings could be obtained, which contributed to the understanding of the distribution of cavitation erosion areas while the orifice was moving. 


\subsection{Effects of groove parameters on characteristics of cavitation erosion}

Reciprocals of the distribution coefficient $k^{-1}$ of the U-shaped two-step groove with different orifice openings were acquired and shown in Fig. 6.

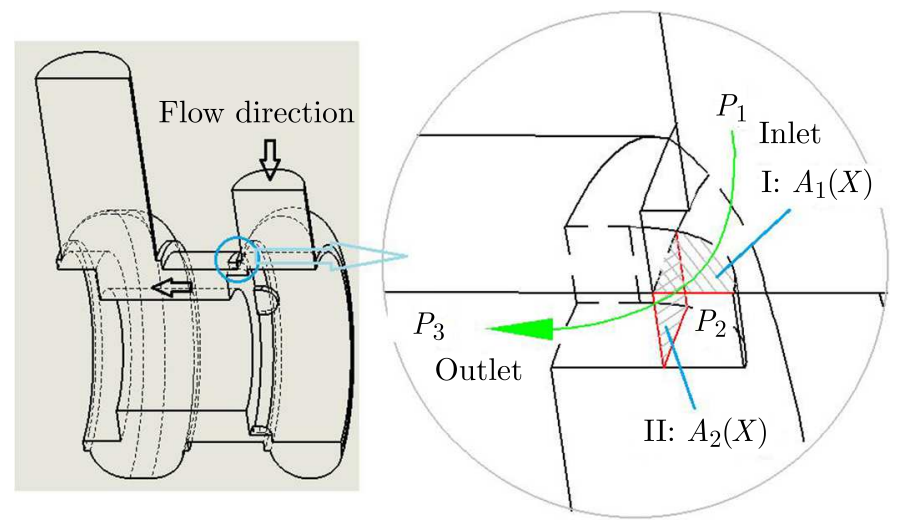

Fig. 6. U-shpaed groove throttling pressure drop distribution coefficient $k^{-1}$

It could be seen from section I and section II that the $k$ of the U-shaped groove and orifice opening $X$ was inversely proportional. Therefore, the reciprocal of the pressure reduction distribution coefficient $k^{-1}$ was taken as a dependent variable and the relationship was demonstrated in Fig. 6. The reciprocal distribution coefficient $k^{-1}$ increases with the increasing valve opening $X$. It can be seen from Eq. (3.11) that $k^{-1}$ has a great influence on the cavitation index $\sigma_{1}$. Moreover, the larger $k^{-1}$, the smaller $\sigma_{1}$, which means that cavitation is not easy to occur.

As observed, $k^{-1}$ dwindled as the groove depth $H$ grew and the gradient showed a decreasing trend, meaning that in different flow sections, the distribution of pressure reduction tended to be stable. So, an increase in $H$ could prevent the concentration of pressure reduction in one flow section. However, the change in the gradient of the $k^{-1}$ curve caused by the change in the radius of the groove $R$ was not evident.

The $k$ of the U-shaped throttle valve was attained through the above analysis, and the inlet pressure of the U-shaped throttle valve $p_{1}$ was set to be $5 \mathrm{MPa}$, the outlet pressure was $0.1 \mathrm{MPa}$, and the vapor pressure $p_{g}$ of room temperature was $1.5 \cdot 10^{4} \mathrm{~Pa}$. So, $\sigma_{1}$ and $\sigma_{2}$ under this condition could be calculated. The characteristics of $\sigma$ with different $H$ were then obtained.

As shown in Fig. 7, $\sigma_{1}$ in section I was higher than its counterpart at a small throttling depth, suggesting that the possibility of cavitation in this position was larger. In other words, the pressure reduction in section I generated under $X_{0}$ increased as $H$ grew; the pressure reduction in section II, therefore, decreased. The overall, though larger $H$ led to growth in $\sigma$ in section I and thus, the risk of cavitation erosion, the pressure reduction in section II declined to some extent. So did the intensity of cavitation, increasing the time needed for $\sigma_{2}$ to reach its peak value.

According to the cavitation erosion index characteristic curve (Fig. 8), when the fluid flowed into and away from section I, it could be seen that $\sigma_{1}\left(A_{1-\text { in }}\right)$ was less than $\sigma_{1}\left(A_{1-\text { out }}\right)$ when the fluid flowed into section I, and $\sigma_{2}\left(A_{1-i n}\right)$ was less than $\sigma_{2}\left(A_{1-\text { out }}\right)$ when the fluid flowed away from section I. With the same flow field in the orifice, section II experienced serious cavitation erosion, and the areas where cavitation occurred under different flow field conditions were remarkably different.

The cavitation in section II was significant when fluid flowed into section I. Despite $\sigma_{1}\left(A_{1-i n}\right)$ being smaller than $\sigma_{1}\left(A_{1-\text { out }}\right)$, the intensity of cavitation erosion when the fluid flowed into section I was generally larger than that when the fluid flowed away.

All the groove parameters in the equation of $\sigma$ were well-defined, so the throttling characteristics could be estimated in the design stage of the groove orifice and cavitation could be 


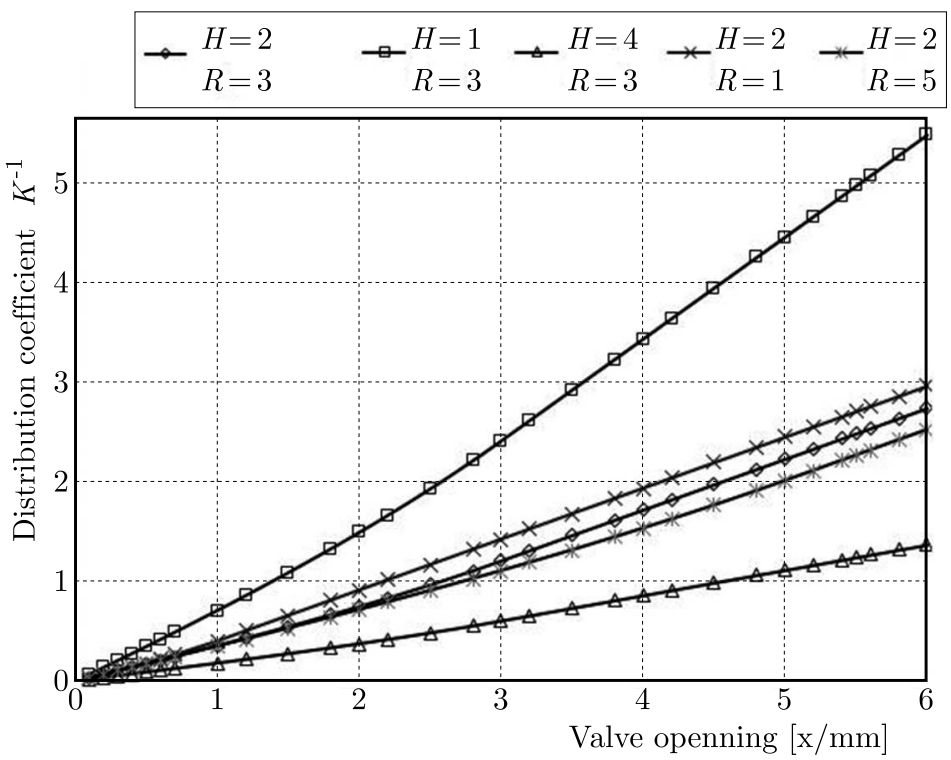

Fig. 7. U-shaped groove $\sigma_{1}$ and $\sigma_{2}$ under different throttling depth $H(R=3 \mathrm{~mm})$

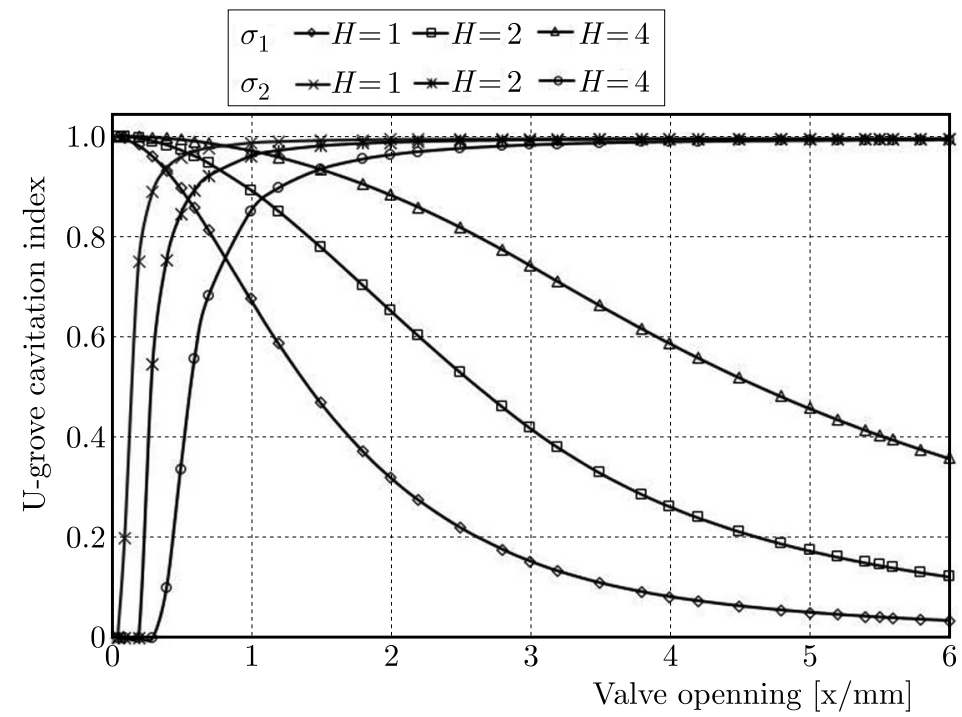

Fig. 8. U-shaped groove $\sigma_{1}$ and $\sigma_{2}$ under flow in and out section I: $A_{1}(X)$

possibly avoided. In addition, the time spent on design and experiment would be reduced, as well as the design cycle of the hydraulic valve.

\section{Conclusions}

Based on the analysis of the flow field of the U-shaped groove orifice, it was found that significant reduction in the fluid pressure occurs near the groove orifice, which would cause serious cavitation erosion.

The cavitation characteristics of the U-shaped two-step throttle valve were obtained in this study. The following analysis showed that a larger $H$ in the U-shaped groove induced a larger cavitation index in section I and had the risk of cavitation erosion. While, the pressure reduction in section II dropped and cavitation was abated, to some extent, which increased the time needed 
for the cavitation index $\sigma_{2}$ to reach its peak value. However, the parameter $R$ did not show a significant impact on cavitation in this study.

Cavitation in section II generally took place when the fluid flowed into section I. Despite the cavitation index $\sigma_{1}\left(A_{1-\text { in }}\right)$ being lower than $\sigma_{1}\left(A_{1-\text { out }}\right)$, the severity of cavitation erosion when the fluid flowed into section I was generally greater than that when it flowed away.

\section{Acknowledgement}

This work was supported in part by the National Natural Science Fund of China, scientific research fund of Nanjing Institute of Technology, practical innovation project of graduate students in Jiangsu Province, Support Fund No. 51505211, 51505212, 11302097. CKJB201901, SJCX20_0700.

\section{References}

1. Amirante R., Distaso E., Tamburrano P., 2014, Experimental and numerical analysis of cavitation in hydraulic proportional directional valves, Energy Conversion and Management, 87, 208-219

2. Chen Y., Li J., Gong Z., Chen X., Lu C., 2019, Large eddy simulation and investigation on the laminar-turbulent transition and turbulence-cavitation interaction in the cavitating flow around hydrofoil, International Journal of Multiphase Multiphase Flow, 112, 300-322

3. Corchuelo O.J., Sото L.L., 2017, Caries prevalence of preschool age children in community homes of the Cauca Valle and related social factors, Revista Odontológica Mexicana, 21, 4, 229-234

4. Favrel A., Pereira J.G. Jr., Landry C., Müller A., Yamaishi K., Avellan F., 2019, Dynamic modal analysis during reduced scale model tests of hydraulic turbines for hydro-acoustic characterization of cavitation flows, Mechanical Systems and Signal Processing, 117, 81-96

5. Ghiji M., Goldsworthy L., Brandner P.A., Garaniya V., Hield P., 2017, Analysis of diesel spray dynamics using a compressible Eulerian/VOF/LES model and microscopic shadowgraphy, Fuel, 188, 352-366

6. Gnanaskandan A., Mahesh K., 2016, Large Eddy Simulation of the transition from sheet to cloud cavitation over a wedge, International Journal of Multiphase Flow, 83, 86-102

7. Han M., Liu Y., Wu D., Zhao X., Tan H., 2017, A numerical investigation in characteristics of flow force under cavitation state inside the water hydraulic poppet valves, International Journal of Heat and Mass Transfer, 111, 1-16

8. Jiang G., Zhang Y., Wen H., Xiao G., 2015, Study of the generated density of cavitation inside diesel nozzle using different fuels and nozzles, Energy Conversion and Management, 103, $208-217$

9. Karrholm F.P., Weller H., Nordin N., 2007, Modelling injector flow including cavitation effects for diesel applications, Proceedings of ASME/JSME 5th Joint Fluids Engineering Conference: Fluids Engineering Division Summer Meeting, 2, 465-474

10. Kiм H., Kiм S., 2019, Optimization of pressure relief valve for pipeline system under transient induced cavitation condition, Urban Water Journal, 16, 10, 718-726

11. Koukouvinis P., Naseri H., Gavaises M., 2017, Performance of turbulence and cavitation models in prediction of incipient and developed cavitation, International Journal of Engine Research, 18, $4,333-350$

12. Lee M.G., Lim C.S., Han S.H., 2016, Shape design of the bottom plug used in a 3-way reversing valve to minimize the cavitation effect, International Journal of Precision Engineering and Manufacturing, 17, 3, 401-406

13. Liu W., Kang Y., Wang X., LiU Q., Fang Z., 2020, Integrated CFD-aided theoretical demonstration of cavitation modulation in self-sustained oscillating jets, Applied Mathematical Modelling, $\mathbf{7 9}, 521-543$ 
14. Liu X., Xu H., Li B., Sun F., 2019, Numerical analysis for unsteady cavitation characteristics in throttle valve, Journal of Vibration and Shock, 38, 3, 89-95

15. PožAr T., Pirc Ž., Susič E., PetkovšEk R., 2020, Simplified detection of cavitation threshold in control valves, Applied Acoustics, 165, 107320

16. Šarc A., Stepišnik-Perdih T., Petkovšek M., Dular M., 2017, The issue of cavitation number value in studies of water treatment by hydrodynamic cavitation, Ultrasonics Sonochemistry, 34, 51-59

17. Sun L., Guo P., Luo X., 2020, Numerical investigation on inter-blade cavitation vortex in a Franics turbine, Renewable Energy, 158, 64-74

18. Yaghoubi H., Madani S.A.H., Alizadeh M., 2018, Numerical study on cavitation in a globe control valve with different numbers of anti-cavitation trims, Journal of Central South University, 25, 11, 2677-2687

Manuscript received March 9, 2021; accepted for print May 13, 2021 